\title{
Genomic characterization of treatment-associated small cell neuroendocrine carcinoma of the prostate
}

\author{
Ivan de Kouchkovsky ${ }^{1}$, David A. Quigley ${ }^{2}$, Eric J. Small ${ }^{1}$, Rahul Aggarwal ${ }^{1}$ \\ 'Department of Medicine, University of California San Francisco, San Francisco, CA 94158, USA. \\ ${ }^{2}$ Department of Urology, University of California San Francisco, San Francisco, CA 94143, USA.
}

Correspondence to: Dr. Ivan de Kouchkovsky, Department of Medicine, University of California San Francisco, 550 16th Street, Box 3211, San Francisco, CA 94158-3211, USA. E-mail: Ivan.deKouchkovsky@ucsf.edu

How to cite this article: de Kouchkovsky I, Quigley DA, Small EJ, Aggarwal R. Genomic characterization of treatment-associated small cell neuroendocrine carcinoma of the prostate. J Transl Genet Genom 2021;5:265-77.

https://dx.doi.org/10.20517/jtgg.2021.32

Received: 11 Jun 2021 First Decision: 6 Jul 2021 Revised: 27 Jul 2021 Accepted: 3 Aug 2021 First online: 3 Aug 2021

Academic Editor: Sanjay Gupta Copy Editor: Xi-Jun Chen Production Editor: Xi-Jun Chen

\begin{abstract}
Treatment-associated small cell neuroendocrine carcinoma of the prostate (t-SCNC) is an aggressive prostate cancer variant with rising incidence. Although morphologically similar to de novo small cell prostate cancer, t-SCNC is thought to emerge from metastatic castration-resistant prostate cancer (mCRPC) under the selective pressure of prolonged AR-targeted therapies. $\mathrm{t}-\mathrm{SCNC}$ is associated with a distinct transcriptional landscape, characterized by the upregulation of stem cell-associated and neuronal programs (e.g., SOX2, N-MYC, FOXA2) and decreased canonical AR signaling. In addition, as with other neuroendocrine carcinomas, RB1 loss and inactivating TP53 mutations are key genomic hallmarks of t-SCNC. Nevertheless, despite their histologic, molecular, and clinical differences, there is a striking degree of genomic overlap between t-SCNC and its adenocarcinoma counterpart. This finding underscores the clonal evolution of t-SCNC from $\mathrm{mCRPC}$, as well as the importance of epigenetic mechanisms in regulating tumor phenotype. In this review, we summarize the key genomic, transcriptional, and epigenetic features of t-SCNC and discuss how recent advances in our understanding of molecular drivers of t$\mathrm{SCNC}$ have contributed to improving the diagnosis and treatment of this aggressive disease.
\end{abstract}

Keywords: Castration-resistant prostate cancer, epigenetics, lineage plasticity, molecular genetics, neuroendocrine prostate cancer, small cell carcinoma, prostate 


\section{INTRODUCTION}

Although novel androgen receptor (AR) targeted therapies have contributed to a recent decline in prostate cancer (PC) mortality ${ }^{[1]}$, metastatic castration-resistant prostate cancer (mCRPC) remains a lethal disease with over 30,000 yearly deaths in the United States alone ${ }^{[2]}$. While most cases of PC initially respond to ARtargeted therapies, prolonged treatment invariably leads to the development of castration-resistant disease. Resistance to AR-targeted therapy can arise in the setting of upregulated AR signaling, often driven by genomic alterations of the AR gene, enhancer, or co-activators. Lineage plasticity constitutes a distinct mechanism of resistance to AR-targeted therapy, whereby selective treatment pressure induces a phenotypic switch from an AR-driven adenocarcinoma to an AR-independent variant - morphologically similar to the rare but highly aggressive de novo small cell prostate cancer. This treatment-emergent variant can present as pure or mixed small cell carcinoma and has been variously labeled neuroendocrine ${ }^{[3]}$ or aggressive variant $^{[4]}$ prostate cancer. We refer to this entity as "treatment-associated small cell neuroendocrine prostate cancer" or t-SCNC, recognizing both its similarity to de novo small cell prostate cancer and its emergence following AR targeted therapy.

t-SCNC is an aggressive PC variant characterized by increased resistance to conventional AR-targeted therapies ${ }^{[5]}$. The optimal treatment of $\mathrm{t}$-SCNC has not been established, and although platinum-based chemotherapy regimens are commonly used, treatment responses are typically short-lived, and prognosis remains poor ${ }^{[4,6]}$. Recent advances in our understanding of the genomic and epigenetic features of t-SCNC have improved our ability to identify patients with t-SCNC while simultaneously bringing into focus a spectrum of aggressive tumor phenotypes with varying levels of AR independence and neuroendocrine differentiation. In addition, improved characterization of the underlying molecular drivers of t-SCNC has also helped to identify potential therapeutic targets in a disease with otherwise limited treatment options.

In this review, we explore the genomic, transcriptional, and epigenetic landscape of t-SCNC. We discuss important genetic drivers of lineage plasticity and some of the mechanisms through which genomic alterations and epigenetic reprogramming contribute to the emergence of t-SCNC. Finally, we review how recent advances in our understanding of t-SCNC have contributed to the development of potential new therapeutic strategies for this aggressive disease.

\section{DIAGNOSIS AND CLINICAL FEATURES}

Small cell neuroendocrine carcinoma (SCNC) of the prostate is rarely present at the time of initial diagnosis $(<2 \% \text { of all PC })^{[7]}$ and more commonly arises in patients with a history of prostatic adenocarcinoma and prior antiandrogen therapy (i.e., t-SCNC) ${ }^{[8,9]}$. Historically thought of as a rare $\mathrm{PC}$ variant ${ }^{[10]}$, an analysis of contemporary series suggests that t-SCNC occurs in as many as $20 \%$ of $\mathrm{mCRPC}$ patients ${ }^{[11]}$. This rising incidence may in part be due to the increasing and earlier use of more intensive AR-targeted therapies, but also likely reflects an ascertainment bias as biopsies of metastatic disease become more common. Traditionally, the diagnosis of SCNC has required the presence of small cell histology on biopsy, along with low AR expression and positive staining for neuroendocrine markers such as chromogranin A or synaptophysin. In more recent years, however, improved molecular profiling has led to the recognition of a subset of patients with aggressive PC lacking small cell histology but with molecular and clinical features of $\mathrm{t}-\mathrm{SCNC} \mathrm{C}^{[12]}$.

Clinically, t-SCNC is associated with significantly shorter survival and decreased response to AR-targeted therapies $^{[5,11]}$. Therefore, a high clinical index of suspicion and prompt initiation of chemotherapy is often required to impact the natural history of this disease. Unfortunately, no single clinical feature can be used to reliably distinguish t-SCNC patients from MCRPC patients with adenocarcinoma (hereafter referred to as 
mCRPC-adenocarcinoma). The presence of visceral or predominantly lytic bone metastases, bulky tumors, low prostate-specific antigen levels relative to tumor burden, and short response to androgen deprivation therapy $(\mathrm{ADT})$ have been used to select patients for platinum-based chemotherapy ${ }^{[4]}$. However, the sensitivity and specificity of these clinical features have not been established. Our group has demonstrated that the combined finding of a serum neuron-specific enolase concentration $>6.05 \mathrm{ng} / \mathrm{mL}$ and chromogranin A level $>3.1 \mathrm{ng} / \mathrm{mL}$ showed high sensitivity and negative predictive value (95\% and $98 \%$, respectively), but low specificity (50\%) and positive predictive value (22\%) for the finding of t-SCNC on biopsy. Although commonly seen at the time of t-SCNC diagnosis, the presence of liver metastases does not predict histology ${ }^{[11]}$.

\section{GENOMIC ALTERATIONS IN T-SCNC}

Unlike other small cell neuroendocrine carcinomas and their non-small cell histologic counterparts ${ }^{[13]}$, there is a surprising overlap in the spectrum of genomic alterations seen in t-SCNC and mCRPCadenocarcinoma. Similar numbers of single nucleotide and copy number variants are observed across tSCNC and mCRPC-adenocarcinoma specimens ${ }^{[3,14]}$, and most of the common PC alterations (such as ERG gene rearrangements and PTEN deletions) are detected in similar frequencies across the two histologic subtypes $^{[15-17]}$. This genomic overlap supports a divergent model of clonal evolution, whereby t-SCNC arises through transdifferentiation of an MCRPC-adenocarcinoma subclone - rather than treatment selection of a pre-existing independent neuroendocrine subclone. The paucity of genomic differences between mCRPCadenocarcinoma and t-SCNC also underscores the potential role of epigenetic reprogramming in mediating lineage plasticity and establishing tumor phenotype, discussed below. In the context of this broad genomic overlap, however, the few genomic differences that have been described across the two histologic subtypes provide important insights into the pathogenesis of t-SCNC.

RB1 loss and inactivating TP53 mutations have long been implicated in cancer development through the activation of E2F transcription factors required for cell cycle progression and downregulation of proapoptotic genes, respectively ${ }^{[18,19]}$. Among PC patients, the incidence of these alterations increases as the disease progresses from non-metastatic adenocarcinoma to $\mathrm{mCRPC}^{[17]}$. Heterogeneous levels of RB1 depletion within individual metastatic biopsies further suggest that $R B 1$ loss constitutes a late subclonal event in $\mathrm{mCRPC}^{[20]}$. Both TP53 mutations and RB1 loss are significantly enriched in patients with t-SCNC: Whole-exome sequencing of $81 \mathrm{mCRPC}$ samples (including $30 \mathrm{t}$-SCNC tumors) thus detected RB1 loss in $70 \%$ of $\mathrm{t}$-SCNC tumors, compared to only $32 \%$ of mCRPC-adenocarcinoma samples $(P=0.003)^{[3]}$. In another cohort of $101 \mathrm{mCRPC}$ patients, RB1 loss was present in all five t-SCNC samples (with the biallelic loss occurring in $60 \%$ of cases), compared to only $9.4 \%$ of mCRPC-adenocarcinoma tumors ${ }^{[14]}$. TP53 alterations, which are found in approximately $30 \%$ of mCRPC-adenocarcinomas, are detected in up to twothirds of t-SCNC cases $(P=0.043)^{[3,14,21]}$. Finally, combined TP53 alteration and RB1 loss (seen in only $4 \%$ $5 \%$ of $\mathrm{mCRPC})^{[15,21]}$ have been reported in more than $50 \%$ of $\mathrm{t}-\mathrm{SCNC}^{[3]}$.

Although RB1 loss and TP53 mutations constitute key genomic hallmarks of t-SCNC, these alterations alone are not sufficient to drive neuroendocrine differentiation or to make a diagnosis of t-SCNC. Indeed, persistent adenocarcinoma phenotype can still be seen in a subset of TP53/RB1 deficient mCRPC samples $^{[21]}$. Similarly, among patients treated with neoadjuvant AR-targeted therapy, the presence of an RB1 loss predicts increased tumor proliferation and treatment resistance but is not itself associated with the expression of neuroendocrine markers ${ }^{[22]}$. Rather than directly inducing neuroendocrine differentiation, RB1 loss and TP53 alterations more likely cooperate to promote lineage plasticity - thereby allowing cancer cells to undergo a phenotypic switch following additional genomic and epigenetic events. Consistent with this model, combined RB1/TP53 loss in PTEN-deficient mouse models of PC has been shown to induce 
lineage plasticity and resistance to AR-targeted therapies through EZH2-dependent epigenetic reprogramming and upregulation of the "stemness" transcriptional factor $S O X 2^{[23-26]}$. Similarly, combined $R B 1 / T P 53$ loss in patient-derived organoids has been shown to synergistically alter chromatin accessibility, promoting expression of stemness transcriptional programs while downregulating prostatic adenocarcinoma and epithelial differentiation program ${ }^{[27]}$. Although the exact sequence of events leading to the emergence of t-SCNC has not been established, these findings suggest that early genomic events such as $R B 1$ loss and inactivating TP53 mutations may induce lineage plasticity, with additional genetic and epigenetic factors leading to subsequent neuroendocrine differentiation and increased cellular proliferation $^{[28]}$.

In addition to the increased frequency of $R B 1$ loss and TP53 mutations that characterize t-SCNC compared with $\mathrm{mCRPC}$-adenocarcinoma, $\mathrm{t}-\mathrm{SCNC}$ is also associated with a paucity of genomic alterations involving the canonical AR signaling pathway, such as amplifications or activating point mutations of the $A R$ gene locus ${ }^{[3,14,22]}$. Whole-genome sequencing similarly shows a relative decrease in the incidence of $A R$ enhancer amplifications among t-SCNC patients compared to those with mCRPC-adenocarcinoma ${ }^{[14]}$. Interestingly, although the overall incidence of mutations involving the $A R$-coregulator Forkhead box A1 (FOXA1) is similar across t-SCNC and mCRPC-adenocarcinoma, FOXA1 R219 mutations are significantly enriched in $\mathrm{t}$-SCNC and have been associated with upregulation of epithelial-mesenchymal-transition (EMT) $\operatorname{program}^{[30]}$. Key genomic features of t-SCNC are summarized in Table 1, and the role of FOXA1 in lineage plasticity is discussed in greater length below.

\section{TRANSCRIPTIONAL PROFILE OF T-SCNC}

Despite its genomic overlap with mCRPC-adenocarcinoma, t-SCNC is associated with a distinct transcriptional profile characterized by the upregulation of stem cell-associated and neuronal programs $s^{[3,1,3,3,32]}$. Consistent with the increased incidence of $R B 1$ loss at the genomic level, transcriptional targets of the $\mathrm{E} 2 \mathrm{~F}$ transcription factor $1\left(\mathrm{E}_{2} \mathrm{~F} 1\right)$ - negatively regulated by the retinoblastoma protein - are among the topmost preferentially expressed genes in $\mathrm{t}-\mathrm{SCNC}^{[11]}$. On the other hand, genes related to the $\mathrm{NOTCH}$ signaling pathway, androgen response, and AR activity are frequently downregulated in t-SCNC relative to $\mathrm{mCRPC}$-adenocarcinoma ${ }^{[3,1,1,14]}$. Distinct from de novo SCNC, and somewhat surprisingly, persistent nuclear AR expression can still be observed in a subset of t-SCNC tumors despite the downregulation of canonical AR transcriptional programs ${ }^{[11]}$. This finding highlights the likely role of epigenetic modification of AR signaling and the presence of alternative, non-canonical AR transcriptional programs in t-SCNC.

SRY-related HMG-box gene 2 ( $\mathrm{SOX}_{2}$ ) is a key mediator of RB1 loss/TP53 inactivation-induced lineage plasticity and has been identified as a master regulator in $\mathrm{t}-\mathrm{SCNC} \mathrm{C}^{[, 1,1,14]} \mathrm{SOX} 2$ serves to maintain pluripotency in human embryonic stem cells ${ }^{[33]}$ and is well known for its ability to reprogram somatic cells into induced pluripotent stem cells when combined with OCT4, KLF4 and $\mathrm{CMYC}^{[34]}$. In the normal prostate, SOX2 expression is restricted to basal epithelial cells. It is repressed by canonical AR signaling ${ }^{[35-37]}$ and conversely upregulated by AR signaling inhibitors ${ }^{[38]}$. SOX2 upregulation in prostate cancer has been associated with a poorly-differentiated stem-cell-like phenotype ${ }^{[35]}$ and is necessary for castration-induced neuroendocrine differentiation in both $P T E N$ null ${ }^{[39]}$ and $R B 1 / T P 3$ null ${ }^{[23]}$ models of prostate cancer. In line with its role in normal human development, $\mathrm{SOX}_{2}$ expression in prostate cancer is thought to contribute to lineage plasticity by promoting the expression of pluripotency genes and repressing master regulators of adenocarcinoma lineage such as FOXA $1^{[40]}$. The exact mechanism through which SOX2 regulates transcription has not been elucidated but, interestingly, does not appear to occur through direct DNA binding. However, SOX2 overexpression induces a dramatic shift in histone $\mathrm{H} 3$ methylation status, 
Table 1. Summary of key genomic alterations associated with t-SCNC

\begin{tabular}{|c|c|c|}
\hline Genes & Summary of findings & Comments \\
\hline RB1/TP53 & $\begin{array}{l}\text { RB1 loss and inactivating TP53 mutations are key genomic hallmarks of } t-S C N C \text {. Their frequency } \\
\text { increases as PC progresses from primary adenocarcinoma to mCRPC-adenocarcinoma. Both } \\
\text { alterations are further enriched in t-SCNC }{ }^{[3,11]}\end{array}$ & $\begin{array}{l}\text { Combined RB1 loss and inactivating TP53 mutations have been implicated with ADT resistance } \\
\text { and lineage plasticity through EZH2-dependent epigenetic reprogramming and upregulation of } \\
\text { the "stemness" transcriptional factor SOX2 }\end{array}$ \\
\hline $\begin{array}{l}A R / A R \text { enhancer/ } \\
\text { AR co-activators }\end{array}$ & $\begin{array}{l}\text { t-SCNC is associated with a relative paucity of genomic alterations (e.g., amplifications, } \\
\left.\text { activating point mutations) involving the } A R \text { gene locus and } A R \text { enhancer }{ }^{3,14}\right]\end{array}$ & $\begin{array}{l}\text { Specific R219 mutations in the AR coactivator and pioneer transcription factor FOXA1 have been } \\
\text { associated with upregulation of EMT programs and t-SCNC } C^{[30]}\end{array}$ \\
\hline SETD2, CYLD & Loss of SETD2 and CYLD has also been associated with t-SCNC ${ }^{[3]}$ & $\begin{array}{l}\text { Genomic loss of } C Y L D \text { is detected in up to half of } \mathrm{t}-\mathrm{SCNC} \text { cases and associated with decreased } \\
\text { canonical AR signaling }\end{array}$ \\
\hline
\end{tabular}

t-SCNC: Treatment-associated small cell neuroendocrine prostate cancer; PC: prostate cancer; mCRPC: metastatic castration-resistant prostate cancer; ADT: androgen deprivation therapy; EZH2: enhancer of zeste homolog 2; AR: androgen receptor; EMT: epithelial-mesenchymal-transition.

mediated in part by lysine-specific demethylase 1 (LSD1), thereby altering chromatin structure and accessibility ${ }^{[40]}$.

MYC (encoded by the MYCN proto-oncogene) is another key transcription factor upregulated in t-SCNC. In normal human development, N-MYC promotes the proliferation of neuronal progenitor cells and plays a critical role in organogenesis. MYCN upregulation has been implicated in the development of various malignancies and is associated with shortened overall survival in prostate cancer patients ${ }^{[1]}$. MYCN expression is upregulated in RB1 deficient LNCaP cell lines $^{[2]]}$ and in $\mathrm{t}-\mathrm{SCNC}$ samples ${ }^{[42]}$ and is thought to play a direct causal role in the emergence of neuroendocrine differentiation. Indeed, $\mathrm{N}-\mathrm{MYC}$ has been shown to cooperate with EZH2 to downregulate AR transcriptional programs, and forced MYCN expression in PTEN deficient PC xenografts recapitulates a poorly-differentiated carcinoma with variable AR expression levels and epithelial-mesenchymal transition phenotype ${ }^{[42]}$. MYCN expression has also been implicated in increased resistance to AR-targeted therapy, in part through upregulation of DNA damage response pathway genes such as $A T M^{[2-44]}$. Interestingly, N-MYC transcriptional activity appears to be modulated by circulating androgen levels. Thus, following androgen depletion, a shift in the NMYC cistrome (i.e., genome-wide binding sites) towards neural development and lineage pathways genes (e.g., SOX2, CHGA) has been described ${ }^{\left[{ }^{22]}\right]}$. This mechanism offers a potential link between exposure to androgen deprivation therapy and the emergence of t-SCNC.

Forkhead box A1 (encoded by the FOXA1 gene) is a pioneer transcription factor and important AR co-regulator. It is the third most commonly altered gene in prostate cancer, with gene rearrangements or point mutations detected in up to a third of patients with $\mathrm{mCRPC}^{[3,45]}$. Canonical FOXA1 signaling maintains epithelial differentiation in prostate and prostate cancer cells, in part through the transcriptional suppression of interleukin-8 - which has itself been implicated in neuroendocrine differentiation via upregulation of enolase 2 expression and the MAPK/ERK pathway ${ }^{[46]}$. Conversely, FOXA1 knockdown in LNCaP prostate adenocarcinoma cells induces neuroendocrine differentiation, and FOXA1 gene expression is significantly lower among t-SCNC samples when compared to prostatic adenocarcinoma ${ }^{[46]}$. However, the exact role of FOXA1 signaling in lineage plasticity and neuroendocrine differentiation has not been fully established, as some evidence suggests that FOXA1 reprogramming - rather than downregulation - contributes to the emergence of t-SCNC. Mutations in 
the R219 hotspot of the forkhead domain (enriched in t-SCNC) have thus been associated with increased FOXA1 binding to non-canonical sites and activation of mesenchymal and neuroendocrine transcriptional programs. Although R219 mutations are detected only in a small subset of $\mathrm{t}-\mathrm{SCNC}^{[30]}$, genome-wide profiling of wild-type FOXA1-binding sites in patient-derived xenografts similarly shows reprogramming of FOXA1 signaling in t-SCNC - with novel binding sites identified near regions of neuronal and stemnessassociated genes ${ }^{[47]}$. Thus despite decreased transcriptional expression, persistent non-canonical FOXA1 signaling may still contribute to neuroendocrine differentiation in t-SCNC.

FOXA2 is another lineage-specific pioneer transcription factor implicated in the emergence of t-SCNC. Unlike FOXA1, FOXA2 expression in the normal adult prostate is restricted to basal epithelial cells ${ }^{[48]}$. Its putative role in the development of t-SCNC was first suggested by the finding of FOXA2 upregulation across various mouse models of $\mathrm{t}-\mathrm{SCNC}^{[48-50]}$. Subsequent work has demonstrated that FOXA2 expression is significantly enriched in t-SCNC patient samples, with strong staining by immunohistochemistry in up to $75 \%$ of t-SCNC tumors, compared with only $4 \%$ of adenocarcinoma samples ${ }^{[51]}$. A more recent transcriptional analysis of mCRPC biopsies has identified FOXA2 as one of the main master regulators preferentially expressed in t-SCNC ${ }^{[11]}$. Upregulation of FOXA2 in t-SCNC appears to be mediated in part by the removal of repressive histone methylation marks in the FOXA2 promoter region ${ }^{[52]}$. The resulting increase in FOXA2 expression, in turn, has been shown to cooperate with hypoxia-inducible factor 1 alpha $\left(\mathrm{HIF}_{1}-\alpha\right)$ to upregulate neuronal programs and specific HIF1 alpha targets genes (HES6, SOX9, and $K D M 3 A)$ under hypoxic conditions ${ }^{[53]}$.

The ONECUT2 master regulator has surfaced in recent years as another key transcriptional regulator of lineage plasticity and mediator of hypoxia-induced neuroendocrine differentiation ${ }^{[5,55]}$. Through pan-cancer mRNA abundance analyses of poorly-differentiated neuroendocrine tumors, ONECUT2 was identified as one of 9 transcription factors (along with ASCL1, INSM1, PROX1, SIX2, MYT1, and MYT1L) differentially upregulated in neuroendocrine tumors compared to their non-neuroendocrine counterpart. In PC specifically, ONECUT2 expression has been shown to be upregulated in prostatic adenocarcinoma (compared with benign prostate tissue) and is associated with an increased risk of biochemical recurrence following primary local therapy ${ }^{[54]}$. ONECUT2 is further upregulated as PC progresses from primary adenocarcinoma to $\mathrm{mCRPC}$ and from mCRPC-adenocarcinoma to t-SCNC, where the highest expression levels are seen ${ }^{[55]}$. In addition to promoting cell cycle-related transcriptional programs (including targets of the E2F transcription factors), ONECUT2 also appears to be a key regulator of hypoxia-induced gene expression and promotes angiogenesis and cellular proliferation under hypoxic conditions ${ }^{[55]}$. Other work has similarly identified ONECUT2 to be a key regulator of t-SCNC through downregulation of AR and FOXA1 transcriptional program and upregulation of neuronal and stem-cell programs ${ }^{[54]}$. The key transcriptional features of t-SCNC are summarized in Table 2.

\section{EPIGENETIC DRIVERS OF T-SCNC}

The paucity of genomic differences observed across mCRPC-adenocarcinoma and t-SCNC underscores the role of epigenetic changes in driving tumor phenotype and lineage plasticity. Indeed, targeted bisulfite sequencing of $\mathrm{CpG}$ methylation status has shown a high degree of concordance between DNA methylation and gene expression levels across t-SCNC and mCRPC-adenocarcinoma ${ }^{[3,56]}$. At the genome-wide level, bisulfite sequencing demonstrates stark differences in the DNA methylation landscapes of t-SCNC and mCRPC-adenocarcinoma tumor samples ${ }^{[2,57}$. Along with DNA methylation, post-translational histone modifications play an important role in regulating chromatin structure and have also been implicated in the emergence of t-SCNC. Genome-wide sequencing of histone acetylation status thus clearly distinguishes neuroendocrine and adenocarcinoma patient-derived xenografts, while genes upregulated in t-SCNC have 
Table 2. Summary of key transcriptional programs associated with t-SCNC

\begin{tabular}{|c|c|c|}
\hline Gene/pathway & Summary of findings & Comments \\
\hline SOX2 & $\begin{array}{l}\text { The "stemness" transcriptional factor SOX } 2 \text { is a key mediator of lineage plasticity in RB1/TP53 depleted } \\
\text { prostate cancer }{ }^{[23]} \text {. SOX2 upregulation promotes the expression of pluripotency genes and represses } \\
\text { master regulators of adenocarcinoma-lineage such as FOXA } 1^{[40]}\end{array}$ & $\begin{array}{l}\text { SOX2 transcriptional regulation does not appear to be mediated by direct DNA binding } \\
\text { but rather through modulation of histone } \mathrm{H} 3 \text { methylation status and chromatin } \\
\text { accessibility }{ }^{[40]}\end{array}$ \\
\hline MYCN & $\begin{array}{l}M Y C N \text { expression is upregulated in } \mathrm{t}-\mathrm{SCNC} \text { samples relative to localized prostate adenocarcinoma and } \\
\text { mCRPC }{ }^{[42]} \text {. N-MYC cooperates with EZH } 2 \text { to downregulate AR transcriptional programs and promotes } \\
\text { resistance to AR-targeted therapy }\end{array}$ & $\begin{array}{l}\text { Androgen depletion induces a shift in the N-MYC cistrome towards neural development } \\
\text { and lineage pathways genes }{ }^{[42]} \text {, thus providing a potential link between exposure to } \\
\text { androgen deprivation therapy and the emergence of t-SCNC }\end{array}$ \\
\hline FOXA1 & $\begin{array}{l}\text { FOXA1 is a pioneer transcription factor, which maintains epithelial differentiation in prostate and } \\
\text { prostate cancer cells. FOXA1 gene expression is significantly lower among t-SCNC samples when } \\
\text { compared to prostatic adenocarcinoma }{ }^{[46]}\end{array}$ & $\begin{array}{l}\text { Mutations in the R219 hotspot of the FOXA1 forkhead domain are associated with t-SCNC } \\
\text { and result in non-canonical FOXA1 signaling and activation of mesenchymal and } \\
\text { neuroendocrine transcriptional programs }\end{array}$ \\
\hline FOXA2 & $\begin{array}{l}\text { FOXA2 is a topmost master regulator of neuroendocrine differentiation, upregulated in up to } 75 \% \text { of t- } \\
\text { SCNC cases staining (vs. only } 4 \% \text { of } \mathrm{mCRPC} \text {-adenocarcinoma samples) }{ }^{[14,51]}\end{array}$ & $\begin{array}{l}\text { FOXA2 upregulation is mediated by the removal of repressive histone methylation marks } \\
\text { in the FOXA2 promoter region }{ }^{[22]}\end{array}$ \\
\hline ONECUT2 & $\begin{array}{l}\text { Several analyses have identified ONECUT2 as a key master regulator of t-SCNC through the promotion } \\
\text { of neuronal, stem-cell, and cell cycle-related transcriptional programs and downregulation of AR and } \\
\text { FOXA1 activity }\end{array}$ & $\begin{array}{l}\text { ONECUT2 has also been implicated in hypoxia-induced neuroendocrine differentiation } \\
\text { through SMAD3 signaling }{ }^{[5]}\end{array}$ \\
\hline
\end{tabular}

t-SCNC: Treatment-associated small cell neuroendocrine prostate cancer; mCRPC: metastatic castration-resistant prostate cancer; EZH2: enhancer of zeste homolog 2; AR: androgen receptor.

been associated with nearby regions of increased histone acetylation ${ }^{[47]}$. Importantly, these dramatic shifts in the epigenetic landscape of t-SCNC are supported by significant changes in tumor metabolism: increased glycolysis - and the resulting production of acetyl-CoA from pyruvate - provides a key substrate for histone acetylation ${ }^{[5,55]}$. Decreased expression of protein kinase $\mathrm{C} \lambda / \mathrm{l}$ and upregulation of phosphoglycerate dehydrogenase (PHDGH) in t-SCNC leads to increased S-adenosyl methionine (SAM) synthesis, which in turn provides methyl groups required for DNA and histone methylation ${ }^{[60]}$. Interestingly, treatment of PC cells with cycloleucine (an inhibitor of SAM synthesis) has been shown to reduced neuroendocrine and basal markers ${ }^{[0]}$. The interplay between tumor metabolism and epigenetic mechanisms suggests a potential for metabolic-directed therapies to reverse lineage plasticity and restore ARdependence in t-SCNC.

In addition to these genome-wide differences, several specific epigenetic pathways and regulators have been implicated in the emergence of t-SCNC [Table 3]. The histone methylase polycomb repressive complex 2 (PRC2) is a key epigenetic regulator of transcription, repressing gene expression through methylation of histone 3 lysine 27 (H3K27me3) on nucleosomes. The histone-lysine N-methyltransferase Enhancer of zeste homolog 2 (EZH2) constitutes the major catalytic enzyme of PRC2 and has emerged in recent years as one of the most important drivers of epigenetic reprogramming in t-SCNC. Indeed, transcriptional profiling of various t-SCNC samples and pre-clinical models have consistently identified $E Z H 2$ as one of the topmost overexpressed epigenetic regulators in t$\mathrm{SCNC}^{[3,61,62]}$. Furthermore, $\mathrm{EZH} 2$ silencing has been shown to prevent neuroendocrine differentiation in several patient-derived xenografts and organoid models of t-SCNC ${ }^{[62,63]}$. However, the exact mechanism through which EZH2 contributes to the development of t-SCNC has not been fully elucidated. EZH2 is a transcriptional target of $\mathrm{E} 2 \mathrm{~F} 1$ and is upregulated in $\mathrm{RB} 1$ depleted tumors ${ }^{[2,62]}$. Androgen deprivation has also been shown to increase EZH2 activity through 
Table 3. Summary of key epigenetic mechanisms and pathways associated with t-SCNC

\begin{tabular}{|c|c|c|}
\hline $\begin{array}{l}\text { Gene/epigenetic } \\
\text { mechanism }\end{array}$ & Summary of findings & Comments \\
\hline DNA methylation & $\begin{array}{l}\text { CpG methylation status shows a high degree of concordance between DNA methylation and gene expression levels } \\
\text { across t-SCNC and mCRPC-adenocarcinoma samples }{ }^{[3,56]}\end{array}$ & $\begin{array}{l}\text { Bisulfite sequencing of cell-free DNA is capable of distinguishing t- } \\
\text { SCNC cases from mCRPC-adenocarcinoma and may provide a novel } \\
\text { non-invasive biomarker of t-SCNC29] }\end{array}$ \\
\hline Histone acetylation & $\begin{array}{l}\text { t-SCNC is associated with increased histone acetylation of regulatory elements near neuronal and stemness genes, } \\
\text { such as CHGA, ASCL1, and SOX2 }\end{array}$ & $\begin{array}{l}\text { Increased rate of glycolysis in } \mathrm{t}-\mathrm{SCNC} \text { results in increased generation } \\
\text { of acetyl-CoA, a key substrate for histone acetylation }\end{array}$ \\
\hline$E Z H 2$ & $\begin{array}{l}\text { The EZH2 histone methyltransferase is one of the topmost overexpressed epigenetic regulators in } \mathrm{t}-\mathrm{SCNC} \mathrm{C}^{[3,61,62]} \text {. } \\
\text { EZH2 cooperates with N-MYC to downregulate AR target gene expression and promote neuronal transcriptional } \\
\text { programs }{ }^{411,42,56]}\end{array}$ & $\begin{array}{l}\text { Androgen deprivation increases EZH2 activity through PKA/CREB } \\
\text { signaling }{ }^{[64]} \text {, offering a potential link between prolonged } \\
\text { antiandrogen therapy and lineage plasticity }\end{array}$ \\
\hline SRRM4 & $\begin{array}{l}\text { SRRM } 4 \text { is upregulated in t-SCNC and accounts for the majority of alternative mRNA splicing events associated with } \\
\mathrm{t}-\mathrm{SCNC} \mathrm{C}^{[65]} \text {. In particular, SRRM } 4 \text { induces an inactive splice variant of REST-1 (a negative master regulator of } \\
\text { neuroendocrine differentiation), which is downregulated in up to } 50 \% \text { of } \mathrm{t}-\mathrm{SCNC} \text { tumors }\end{array}$ & $\begin{array}{l}\text { SRRM } 4 \text { has also been shown to induce a splice variant of the histone } \\
\text { modulator LSD1 (LSD1+8a), which in turn regulates AR signaling and } \\
\text { promotes neuronal gene expression }{ }^{[73]}\end{array}$ \\
\hline
\end{tabular}

t-SCNC: Treatment-associated small cell neuroendocrine prostate cancer; mCRPC: metastatic castration-resistant prostate cancer; EZH2: enhancer of zeste homolog 2; AR: androgen receptor; LSD1: Iysine-specific demethylase 1; SRRM4: serine/arginine-repetitive matrix 4

PKA/CREB signaling ${ }^{[6]}$, suggesting a potential mechanism by which prolonged antiandrogen therapy may promote lineage plasticity. EZH2 has, in turn, been shown to cooperate with N-MYC to downregulate AR target gene expression and promote neuronal transcriptional programs ${ }^{[41,42,56]}$. Conversely, EZH2 silencing in patient-derived t-SCNC models prevents neuroendocrine differentiation and leads to a decrease in global H3K27 methylation status associated with downregulation of stemness and neuronal transcriptional programs ${ }^{[6,63]}$.

The serine/arginine-repetitive matrix 4 (SRRM4) splicing factor is also upregulated in t-SCNC and is estimated to account for the majority of alternative splicing events associated with t-SCNC ${ }^{[65]}$. Pre-clinical models suggest that its activity is enhanced by RB1/TP53 depletion and antiandrogen therapy ${ }^{[65]}$. Genes susceptible to SRRM4-mediated alternative splicing include a variety of other RNA splicing factors and neural-specific histone modifiers such as BHC80 and $L S D 1$, further highlighting the role of epigenetic reprogramming driving lineage plasticity and neuroendocrine differentiation. SRRM4 has also been shown to induce an inactive splice variant of the repressor element-1 silencing transcription factor (REST-1). REST-1 is a negative master regulator of neuroendocrine differentiation, and in its intact form, functions to suppress neuronal transcriptional programs in non-neuronal cells. It is also an important repressor of gene expression in hypoxic conditions ${ }^{[6]}$. Downregulation of REST is seen in up to $50 \%$ of t-SCNC tumors (compared with only $3 \%$ of metastatic prostate adenocarcinoma) $)^{[6]}$ and has been shown to promote epithelial-mesenchymal transition and stemness transcriptional programs (e.g., SOX2, POU3F2 $)^{[32,68]}$.

Although AR independence is considered a hallmark of t-SCNC, persistent AR expression can still be seen in a subset of cases ${ }^{[11]}$. Recent insights suggest a role for epigenetic reprogramming of AR signaling in t-SCNC. Unlike pioneering transcription factors (such as FOXA1), which are able to bind to DNA within areas of condensed chromatin, AR preferentially binds to open chromatin within nucleosome-depleted regions ${ }^{[6]]}$. Epigenetic mechanisms of chromatin 
remodeling and AR recruitment have thus been implicated in modulating AR signaling in t-SCNC. As discussed above, repressive histone methylation marks induced by the cooperative action of N-MYC and EZH-2 downregulate canonical AR transcriptional programs in t-SCNC. The histone modulator LSD1 (also known as KDM1A) similarly regulates AR signaling via both transcriptional repression (through $\mathrm{H}_{3} \mathrm{~K} 4$ and H3K9 demethylation) and coactivation (through histone $\mathrm{H} 3$ phosphorylation) of AR-target genes ${ }^{[70]}$. Increased LSD1 expression, which is associated with $R B 1$ loss in prostate cancer cells ${ }^{[71]}$, has been implicated in lineage plasticity through upregulation of stemness and EMT transcriptional programs, as well as induction of glycolysis-shifted metabolism ${ }^{[72]}$. A t-SCNC-specific splice variant of LSD1 (LSD 1+8a) induced by SRRM-4 and associated with activation of neuronal gene expression was recently described ${ }^{[73]}$.

\section{CLINICAL IMPLICATIONS AND FUTURE DIRECTIONS}

$\mathrm{t}$-SCNC is an aggressive clinical entity with limited treatment options. Establishing a diagnosis of t-SCNC has important therapeutic implications due to the increased resistance to conventional hormonal therapies. Unfortunately, reliable diagnostic biomarkers are still lacking ${ }^{[1]}$. While metastatic biopsies provide important information, they remain both technically challenging and subject to intra-patient heterogeneity ${ }^{[14]}$. Although circulating tumor DNA-based biomarkers have the potential to provide a noninvasive patient-wide assessment of the tumor genome, their utility in this clinical scenario has been limited by the significant genomic overlap between t-SCNC and mCRPC. More recently, advances in epigenetic profiling of CRPC and the observation of stark differences in the DNA methylation landscape between the two histologic subtypes have helped to develop novel epigenetic biomarkers. Bisulfite sequencing of cell-free DNA has thus been shown to distinguish $\mathrm{t}$-SCNC cases from mCRPC-adenocarcinoma ${ }^{[29]}$.

Improved molecular characterization of t-SCNC has also highlighted a spectrum of neuroendocrine differentiation across patient metastases (i.e., intra-patient heterogeneity) ${ }^{[14]}$, underscoring the role of continued AR-targeted therapy in patients with t-SCNC. Additionally, the gradient of t-SCNC transcriptional expression observed across t-SCNC samples (i.e., intra-class heterogeneity $)^{[14]}$ suggests a gradual and dynamic process of neuroendocrine differentiation ${ }^{[28]}$; therapeutic strategies targeting the epigenetic mechanisms of lineage plasticity in t-SCNC may offer the potential of reversing neuroendocrine differentiation and restoring AR-dependence. Finally, the development of t-SCNC transcriptional signatures has identified a subset of $\mathrm{mCRPC}$-adenocarcinoma patients with transcriptional features of $\mathrm{t}$ $\mathrm{SCNC}^{[14,21,41]}$, and there is growing evidence to suggest that stemness and t-SCNC transcriptional profiles may predict de novo resistance to ADT and AR-targeted therapies in PC lacking t-SCNC histology ${ }^{[3,74,75]}$. Although the optimal management of such patients has not been established, these findings support the increasing use of molecularly defined inclusion criteria for clinical trials of t-SCNC directed therapies. In the future, the diagnosis of t-SCNC may transition from a purely histology-based definition to a composite of histologic and molecular features.

Beyond its role in the diagnosis of t-SCNC, improved molecular characterization of t-SCNC has also helped identify novel therapeutic strategies in t-SCNC. These include both attempts to target increased cellular proliferation and molecular pathways upregulated in t-SCNC, as well as efforts to reverse lineage plasticity and restore AR-dependence. Aurora kinase A is a cell cycle kinase that stabilizes N-MYC and prevents NMYC protein degradation. Early observations of MYCN upregulation in t-SCNC spurred the development of alisertib, an allosteric inhibitor of Aurora kinase A. Although a phase II trial of single-agent alisertib in SCNC failed to meet its primary endpoint of six-month radiographic progression-free survival, exceptional responses were observed in a subset of patients with N-MYC and Aurora A kinase overactivity ${ }^{[76]}$. Aurora kinase inhibitors may thus still play a role in a molecularly selected subset of t-SCNC. Targeting DNA damage response pathways in $M Y C N$-upregulated tumors has also shown promising activity in pre-clinical 
PC models, reversing neuroendocrine differentiation and restoring sensitivity to AR-targeted therapies ${ }^{[43,44,7]}$. A phase 2 study of maintenance olaparib following platinum-based chemotherapy in aggressive variant prostate cancer is currently underway (NCT03263650). The small molecule inhibitor of ONECUT2 CSRM617 has also shown encouraging pre-clinical activity in t-SCNC cell lines and PDX model $^{[54]}$, and indirect targeting of ONECUT2 activity through the hypoxia-activated pro-drug TH-302 is under current clinical investigation in a phase 1b/2 study of mCRPC (NCT00743379). Finally, several trials of drugs targeting epigenetic pathways are underway: bromodomain inhibitors of epigenetic reader BET proteins, such as ZEN-3694 (Zenith Epigenetics), have shown encouraging activity in aggressive variant PC (i.e., with clinical features suggestive of t-SCNC) ${ }^{[78]}$, while $\mathrm{EZH} 2$ inhibitors (e.g., tazemetostat, CPI-1205) have generated promising pre-clinical and early clinical data ${ }^{[42,63,79,80]}$. CPI-1205 (Constellation Pharmaceuticals) is under current investigation in combination with AR-targeted therapy in a phase $1 \mathrm{~b} / 2$ trial of mCRPC patients (NCT03480646).

\section{CONCLUSION}

Phenotypic switch to t-SCNC is an increasingly recognized mechanism of resistance to AR-targeted therapy. Improved molecular profiling has revealed a distinct t-SCNC transcriptional landscape characterized by the upregulation of stem-cell-associated and neuronal programs, as well as decreased canonical AR activity ${ }^{[3,1,1,4,31,32]}$. Nevertheless, despite stark differences in their transcriptional, histologic, and clinical features, there is a surprising degree of genomic overlap between $\mathrm{mCRPC}$-adenocarcinoma and t$\mathrm{SCNC}^{[3,11,14]}$. Furthermore, with a few notable exceptions (e.g., TP53, $R B 1$, and $A R$ ), many gene expression differences observed across the two histologic subtypes are not accounted for by underlying genomic alterations. This finding supports a divergent model of evolution, whereby t-SCNC transdifferentiates from a mCRPC-adenocarcinoma subclone. It also underscores the role of epigenetic mechanisms in driving lineage plasticity and neuroendocrine differentiation. Thus, in addition to the observation of clear genomewide differences in the DNA methylation and histone modification landscapes across the two histologic subtypes $^{[3,47,57]}$, several epigenetic regulators (e.g., EZH2, SRRM4) have recently emerged as key drivers of t$\mathrm{SCNC}^{[6,65]}$. Improved understanding of the molecular underpinnings of t-SCNC carries important implications for the management of this aggressive MCRPC variant: transcriptional and epigenetic signatures have already shown promising results in the diagnosis of t-SCNC and in the development of non-invasive biomarkers. Therapeutic strategies targeting the unique genomic and epigenetic features of $\mathrm{t}$ SCNC are similarly underway, with encouraging pre-clinical and early clinical activity.

\section{DECLARATIONS}

\section{Authors' contributions}

Reviewed literature and wrote the manuscript: de Kouchkovsky I

Provided critical review and editing of the manuscript: Small EJ, Quigley DA, Aggarwal R

\section{Availability of data and materials}

Not applicable.

\section{Financial support and sponsorship}

None.

\section{Conflicts of interest}

Small EJ reports consulting fees from Janssen, Fortis, Teon Therapeutics, Ulragenyx, Beigene, Tolero, honoraria for speaking enagements from Janssen, Johnson and Johnson, and stock in Fortis Therapeutics and Harpoon Therapeutics. Quigley DA reports consulting fees from Varian and Circle Pharma. de Kouchkovsky I and Aggarwal R declared that there are no conflicts of interest. 


\section{Ethical approval and consent to participate}

Not applicable.

\section{Consent for publication}

Not applicable.

\section{Copyright}

(c) The Author(s) 2021.

\section{REFERENCES}

1. Welch HG, Albertsen PC. Reconsidering prostate cancer mortality - the future of PSA screening. N Engl J Med 2020;382:1557-63. DOI PubMed

2. Siegel RL, Miller KD, Jemal A. Cancer statistics, 2019. CA Cancer J Clin 2018;69:7-34. DOI PubMed

3. Beltran H, Prandi D, Mosquera JM, et al. Divergent clonal evolution of castration-resistant neuroendocrine prostate cancer. Nat Med 2016;22:298-305. DOI PubMed PMC

4. Aparicio AM, Harzstark AL, Corn PG, et al. Platinum-based chemotherapy for variant castrate-resistant prostate cancer. Clin Cancer Res 2013;19:3621-30. DOI PubMed PMC

5. Conteduca V, Oromendia C, Eng KW, et al. Clinical features of neuroendocrine prostate cancer. Eur J Cancer 2019;121:7-18. DOI PubMed PMC

6. Spiess PE, Pettaway CA, Vakar-Lopez F, et al. Treatment outcomes of small cell carcinoma of the prostate: a single-center study. Cancer 2007;110:1729-37. DOI PubMed

7. Tětu B, Ro JY, Ayala AG, Johnson DE, Logothetis CJ, Ordonez NG. Small cell carcinoma of the prostate part I a clinicopathologic study of 20 cases. Cancer 1987;59:1803-9. DOI PubMed

8. Epstein JI, Amin MB, Beltran H, et al. Proposed morphologic classification of prostate cancer with neuroendocrine differentiation. Am J Surg Pathol 2014;38:756-67. DOI PubMed PMC

9. Dong B, Miao J, Wang Y, et al. Single-cell analysis supports a luminal-neuroendocrine transdifferentiation in human prostate cancer. Commun Biol 2020;3:778. DOI PubMed PMC

10. Turbat-Herrera EA, Herrera GA, Gore I, Lott RL, Grizzle WE, Bonnin JM. Neuroendocrine differentiation in prostatic carcinomas. A retrospective autopsy study. Arch Pathol Lab Med 1988;112:1100-5. PubMed

11. Aggarwal R, Huang J, Alumkal JJ, et al. Clinical and genomic characterization of treatment-emergent small-cell neuroendocrine prostate cancer: a multi-institutional prospective study. J Clin Oncol 2018;36:2492-503. DOI PubMed PMC

12. Aparicio AM, Shen L, Tapia EL, et al. Combined tumor suppressor defects characterize clinically defined aggressive variant prostate cancers. Clin Cancer Res 2016;22:1520-30. DOI PubMed PMC

13. George J, Lim JS, Jang SJ, et al. Comprehensive genomic profiles of small cell lung cancer. Nature 2015;524:47-53. DOI PubMed PMC

14. Aggarwal RR, Quigley DA, Huang J, et al. Whole-genome and transcriptional analysis of treatment-emergent small-cell neuroendocrine prostate cancer demonstrates intraclass heterogeneity. Mol Cancer Res 2019;17:1235-40. DOI PubMed PMC

15. Quigley DA, Dang HX, Zhao SG, et al. Genomic hallmarks and structural variation in metastatic prostate cancer. Cell 2018;174:75869.e9. DOI PubMed PMC

16. Genome Atlas Research Network. The molecular taxonomy of primary prostate cancer. Cell 2015;163:1011-25. DOI

17. Robinson D, Van Allen EM, Wu YM, et al. Integrative clinical genomics of advanced prostate cancer. Cell 2015;161:1215-28. DOI PubMed PMC

18. Schlereth K, Heyl C, Krampitz AM, et al. Characterization of the p53 cistrome--DNA binding cooperativity dissects p53's tumor suppressor functions. PLoS Genet 2013;9:e1003726. DOI PubMed PMC

19. Macleod KF. The RB tumor suppressor: a gatekeeper to hormone independence in prostate cancer? J Clin Invest 2010;120:4179-82. DOI PubMed PMC

20. Nava Rodrigues D, Casiraghi N, Romanel A, et al. RB1 heterogeneity in advanced metastatic castration-resistant prostate cancer. Clin Cancer Res 2019;25:687-97. DOI PubMed

21. Nyquist MD, Corella A, Coleman I, et al. Combined TP53 and RB1 loss promotes prostate cancer resistance to a spectrum of therapeutics and confers vulnerability to replication stress. Cell Rep 2020;31:107669. DOI PubMed PMC

22. Sowalsky AG, Ye H, Bhasin M, et al. Neoadjuvant-intensive androgen deprivation therapy selects for prostate tumor foci with diverse subclonal oncogenic alterations. Cancer Res 2018;78:4716-30. DOI PubMed PMC

23. Mu P, Zhang Z, Benelli M, et al. SOX2 promotes lineage plasticity and antiandrogen resistance in TP53- and RB1-deficient prostate cancer. Science 2017;355:84-8. DOI PubMed PMC

24. Ku SY, Rosario S, Wang Y, et al. Rb1 and Trp53 cooperate to suppress prostate cancer lineage plasticity, metastasis, and antiandrogen resistance. Science 2017;355:78-83. DOI PubMed PMC

25. Kareta MS, Gorges LL, Hafeez S, et al. Inhibition of pluripotency networks by the Rb tumor suppressor restricts reprogramming and tumorigenesis. Cell Stem Cell 2015;16:39-50. DOI PubMed PMC

26. Soundararajan R, Aparicio AM, Logothetis CJ, Mani SA, Maity SN. Function of tumor suppressors in resistance to antiandrogen 
therapy and luminal epithelial plasticity of aggressive variant neuroendocrine prostate cancers. Front Oncol 2018;8:69. DOI PubMed PMC

27. Park JW, Lee JK, Sheu KM, et al. Reprogramming normal human epithelial tissues to a common, lethal neuroendocrine cancer lineage. Science 2018;362:91-5. DOI PubMed PMC

28. Zou M, Toivanen R, Mitrofanova A, et al. Transdifferentiation as a mechanism of treatment resistance in a mouse model of castrationresistant prostate cancer. Cancer Discov 2017;7:736-49. DOI PubMed PMC

29. Beltran H, Romanel A, Conteduca V, et al. Circulating tumor DNA profile recognizes transformation to castration-resistant neuroendocrine prostate cancer. $J$ Clin Invest 2020;130:1653-68. DOI PubMed PMC

30. Adams EJ, Karthaus WR, Hoover E, et al. FOXA1 mutations alter pioneering activity, differentiation and prostate cancer phenotypes. Nature 2019;571:408-12. DOI PubMed PMC

31. Smith BA, Sokolov A, Uzunangelov V, et al. A basal stem cell signature identifies aggressive prostate cancer phenotypes. Proc Natl Acad Sci U S A 2015;112:E6544-52. DOI PubMed PMC

32. Labrecque MP, Coleman IM, Brown LG, et al. Molecular profiling stratifies diverse phenotypes of treatment-refractory metastatic castration-resistant prostate cancer. J Clin Invest 2019;129:4492-505. DOI PubMed PMC

33. Schaefer T, Lengerke C. SOX2 protein biochemistry in stemness, reprogramming, and cancer: the PI3K/AKT/SOX2 axis and beyond. Oncogene 2020;39:278-92. DOI PubMed PMC

34. Takahashi K, Yamanaka S. Induction of pluripotent stem cells from mouse embryonic and adult fibroblast cultures by defined factors. Cell 2006;126:663-76. DOI PubMed

35. Kregel S, Kiriluk KJ, Rosen AM, et al. Sox2 is an androgen receptor-repressed gene that promotes castration-resistant prostate cancer. PLoS One 2013;8:e53701. DOI PubMed PMC

36. Akamatsu S, Wyatt AW, Lin D, et al. The placental gene PEG10 Promotes progression of neuroendocrine prostate cancer. Cell Rep 2015;12:922-36. DOI PubMed

37. Kim S, Thaper D, Bidnur S, et al. PEG10 is associated with treatment-induced neuroendocrine prostate cancer. J Mol Endocrinol 2019;63:39-49. DOI PubMed

38. Verma S, Shankar E, Kalayci FNC, et al. Androgen deprivation induces transcriptional reprogramming in prostate cancer cells to develop stem cell-like characteristics. Int J Mol Sci 2020;21:9568. DOI PubMed PMC

39. Kwon OJ, Zhang L, Jia D, Xin L. Sox2 is necessary for androgen ablation-induced neuroendocrine differentiation from Pten null Sca$1^{+}$prostate luminal cells. Oncogene 2021;40:203-14. DOI PubMed PMC

40. Li H, Wang L, Li Z, et al. SOX2 has dual functions as a regulator in the progression of neuroendocrine prostate cancer. Lab Invest 2020;100:570-82. DOI PubMed

41. Berger A, Brady NJ, Bareja R, et al. N-Myc-mediated epigenetic reprogramming drives lineage plasticity in advanced prostate cancer. J Clin Invest 2019;129:3924-40. DOI PubMed PMC

42. Dardenne E, Beltran H, Benelli M, et al. N-Myc induces an EZH2-mediated transcriptional program driving neuroendocrine prostate cancer. Cancer Cell 2016;30:563-77. DOI PubMed PMC

43. Yin Y, Xu L, Chang Y, et al. N-Myc promotes therapeutic resistance development of neuroendocrine prostate cancer by differentially regulating miR-421/ATM pathway. Mol Cancer 2019;18:11. DOI PubMed PMC

44. Zhang W, Liu B, Wu W, et al. Targeting the MYCN-PARP-DNA damage response pathway in neuroendocrine prostate cancer. Clin Cancer Res 2018;24:696-707. DOI PubMed PMC

45. Parolia A, Cieslik M, Chu SC, et al. Distinct structural classes of activating FOXA1 alterations in advanced prostate cancer. Nature 2019;571:413-8. DOI PubMed PMC

46. Kim J, Jin H, Zhao JC, et al. FOXA1 inhibits prostate cancer neuroendocrine differentiation. Oncogene 2017;36:4072-80. DOI PubMed PMC

47. Baca SC, Takeda DY, Seo JH, et al. Reprogramming of the FOXA1 cistrome in treatment-emergent neuroendocrine prostate cancer. Nat Commun 2021;12:1979. DOI PubMed PMC

48. Mirosevich J, Gao N, Matusik RJ. Expression of Foxa transcription factors in the developing and adult murine prostate. Prostate 2005;62:339-52. DOI PubMed

49. Lee JK, Phillips JW, Smith BA, et al. N-Myc drives neuroendocrine prostate cancer initiated from human prostate epithelial cells. Cancer Cell 2016;29:536-47. DOI PubMed PMC

50. Chiaverotti T, Couto SS, Donjacour A, et al. Dissociation of epithelial and neuroendocrine carcinoma lineages in the transgenic adenocarcinoma of mouse prostate model of prostate cancer. Am J Pathol 2008;172:236-46. DOI PubMed PMC

51. Park JW, Lee JK, Witte ON, Huang J. FOXA2 is a sensitive and specific marker for small cell neuroendocrine carcinoma of the prostate. Mod Pathol 2017;30:1262-72. DOI PubMed PMC

52. Liu Q, Pang J, Wang LA, et al. Histone demethylase PHF8 drives neuroendocrine prostate cancer progression by epigenetically upregulating FOXA2. J Pathol 2021;253:106-18. DOI PubMed PMC

53. Qi J, Nakayama K, Cardiff RD, et al. Siah2-dependent concerted activity of HIF and FoxA2 regulates formation of neuroendocrine phenotype and neuroendocrine prostate tumors. Cancer Cell 2010;18:23-38. DOI PubMed PMC

54. Rotinen M, You S, Yang J, et al. ONECUT2 is a targetable master regulator of lethal prostate cancer that suppresses the androgen axis. Nat Med 2018;24:1887-98. DOI PubMed PMC

55. Guo H, Ci X, Ahmed M, et al. ONECUT2 is a driver of neuroendocrine prostate cancer. Nat Commun 2019;10:278. DOI PubMed $\mathrm{PMC}$

56. Kleb B, Estécio MR, Zhang J, et al. Differentially methylated genes and androgen receptor re-expression in small cell prostate 
carcinomas. Epigenetics 2016;11:184-93. DOI PubMed PMC

57. Zhao SG, Chen WS, Li H, et al. The DNA methylation landscape of advanced prostate cancer. Nat Genet 2020;52:778-89. DOI PubMed PMC

58. Choi SYC, Ettinger SL, Lin D, et al. Targeting MCT4 to reduce lactic acid secretion and glycolysis for treatment of neuroendocrine prostate cancer. Cancer Med 2018;7:3385-92. DOI PubMed PMC

59. Li W, Cohen A, Sun Y, et al. The role of CD44 in glucose metabolism in prostatic small cell neuroendocrine carcinoma. Mol Cancer Res 2016;14:344-53. DOI PubMed PMC

60. Reina-Campos M, Linares JF, Duran A, et al. Increased serine and one-carbon pathway metabolism by PKC $/ / \mathrm{l}$ deficiency promotes neuroendocrine prostate cancer. Cancer Cell 2019;35:385-400.e9. DOI PubMed PMC

61. Clermont PL, Lin D, Crea F, et al. Polycomb-mediated silencing in neuroendocrine prostate cancer. Clin Epigenetics 2015;7:40. DOI PubMed PMC

62. Puca L, Bareja R, Prandi D, et al. Patient derived organoids to model rare prostate cancer phenotypes. Nat Commun 2018;9:2404. DOI PubMed PMC

63. Shan J, Al-Muftah MA, Al-Kowari MK, et al. Targeting Wnt/EZH2/microRNA-708 signaling pathway inhibits neuroendocrine differentiation in prostate cancer. Cell Death Discov 2019;5:139. DOI PubMed PMC

64. Zhang Y, Zheng D, Zhou T, et al. Androgen deprivation promotes neuroendocrine differentiation and angiogenesis through CREBEZH2-TSP1 pathway in prostate cancers. Nat Commun 2018;9:4080. DOI PubMed PMC

65. Li Y, Donmez N, Sahinalp C, et al. SRRM4 drives neuroendocrine transdifferentiation of prostate adenocarcinoma under androgen receptor pathway inhibition. Eur Urol 2017;71:68-78. DOI PubMed

66. Cavadas MA, Mesnieres M, Crifo B, et al. REST is a hypoxia-responsive transcriptional repressor. Sci Rep 2016;6:31355. DOI PubMed PMC

67. Lapuk AV, Wu C, Wyatt AW, et al. From sequence to molecular pathology, and a mechanism driving the neuroendocrine phenotype in prostate cancer. J Pathol 2012;227:286-97. DOI PubMed PMC

68. Chang YT, Lin TP, Campbell M, et al. REST is a crucial regulator for acquiring EMT-like and stemness phenotypes in hormonerefractory prostate cancer. Sci Rep 2017;7:42795. DOI PubMed PMC

69. Jia L, Berman BP, Jariwala U, et al. Genomic androgen receptor-occupied regions with different functions, defined by histone acetylation, coregulators and transcriptional capacity. PLoS One 2008;3:e3645. DOI PubMed PMC

70. Cai $\mathrm{C}, \mathrm{He} \mathrm{HH}$, Chen $\mathrm{S}$, et al. Androgen receptor gene expression in prostate cancer is directly suppressed by the androgen receptor through recruitment of lysine-specific demethylase 1. Cancer Cell 2011;20:457-71. DOI PubMed PMC

71. Liang Y, Ahmed M, Guo H, et al. LSD1-mediated epigenetic reprogramming drives CENPE expression and prostate cancer progression. Cancer Res 2017;77:5479-90. DOI PubMed

72. Hino S, Kohrogi K, Nakao M. Histone demethylase LSD1 controls the phenotypic plasticity of cancer cells. Cancer Sci 2016;107:1187-92. DOI PubMed PMC

73. Coleman DJ, Sampson DA, Sehrawat A, et al. Alternative splicing of LSD1+8a in neuroendocrine prostate cancer is mediated by SRRM4. Neoplasia 2020;22:253-62. DOI PubMed PMC

74. Alumkal JJ, Sun D, Lu E, et al. Transcriptional profiling identifies an androgen receptor activity-low, stemness program associated with enzalutamide resistance. Proc Natl Acad Sci U S A 2020;117:12315-23. DOI PubMed PMC

75. Zhang D, Park D, Zhong Y, et al. Stem cell and neurogenic gene-expression profiles link prostate basal cells to aggressive prostate cancer. Nat Commun 2016;7:10798. DOI PubMed PMC

76. Beltran H, Oromendia C, Danila DC, et al. A phase II trial of the Aurora Kinase A inhibitor alisertib for patients with castrationresistant and neuroendocrine prostate cancer: efficacy and biomarkers. Clin Cancer Res 2019;25:43-51. DOI PubMed PMC

77. Liu B, Li L, Yang G, et al. PARP inhibition suppresses GR-MYCN-CDK5-RB1-E2F1 signaling and neuroendocrine differentiation in castration-resistant prostate cancer. Clin Cancer Res 2019;25:6839-51. DOI PubMed PMC

78. Aggarwal RR, Schweizer MT, Nanus DM, et al. A phase Ib/IIa study of the pan-BET inhibitor ZEN-3694 in combination with enzalutamide in patients with metastatic castration-resistant prostate cancer. Clin Cancer Res 2020;26:5338-47. DOI PubMed PMC

79. Knutson SK, Wigle TJ, Warholic NM, et al. A selective inhibitor of EZH2 blocks H3K27 methylation and kills mutant lymphoma cells. Nat Chem Biol 2012;8:890-6. DOI PubMed

80. McCabe MT, Ott HM, Ganji G, et al. EZH2 inhibition as a therapeutic strategy for lymphoma with EZH2-activating mutations. Nature 2012;492:108-12. DOI PubMed 